\title{
Joint State and Parameter Estimation For Biochemical Dynamic Pathways With Iterative Extended Kalman Filter: Comparison With Dual State and Parameter Estimation
}

\author{
Zhong Ji ${ }^{\mathrm{a}, \mathrm{b} *}$, Martin Brown ${ }^{\mathrm{b}}$ \\ ${ }^{a}$ Test Center, College of Mechanical Engineering, Chongqing University, Chongqing, 400030, China \\ ${ }^{b}$ School of Electrical \& Electronic Engineering, University of Manchester, Manchester, UK
}

\begin{abstract}
A biochemical dynamic pathway is usually modeled as a nonlinear system described by a set of nonlinear ODEs. In most cases, only partial states can be measured. Moreover, the system parameters, reaction rates, may be unknown or poorly known. Therefore, it is of significance to estimate the states and parameters, for analyzing the biochemical dynamic pathway. Due to the limitation of some traditional parameter estimation approaches, it is natural to choose sequential methods such as extended Kalman filter to do the parameter estimation for biochemical dynamic pathways. In this paper, dual/joint state and parameter estimation with iterative extended Kalman filter (EKF) are investigated to obtain state and parameter estimates for a biochemical pathway simultaneously. The simulated results between two methods are compared to show the validity of parameter estimation for a biochemical dynamic pathway. It has shown that, for the nonlinear biochemical system, the joint state and parameter estimation with EKF, can give desirable convergence and estimation performance.
\end{abstract}

\section{INTRODUCTION}

Systems biology learns about a biological system by analyzing the mathematical models expressed by a set of ODEs of the system. In most cases, only a restricted number of concentrations and reaction rates can be measured/known for a biochemical network, and many of the parameters describing the dynamics of these pathways are unknown or poorly known. Therefore it is worth investigating how to estimate the unknown parameters in the mathematical models just from the partially noisy measured data. There are indirect and direct methods for parameter estimation. The advantage of using the indirect methods is that there are a number of effective parameter estimation algorithms available, e.g., least squares(LS), multistage least squares (MLS), and generalized least squares(GLS). And the existing algorithms used to directly estimate continuous model parameters, such as nonlinear least squares(NLS), system reference adaptive model(SRAM), prediction error(PE), and maximum likelihood(ML) algorithms, are all very time consuming [1].

The common used estimation method, least square estimation, is good for functions that can be linearized and generally best used with data sets containing complete data. Least-square estimators assume that the noise contaminating the data is of zero mean, which yields an unbiased parameter estimate. If the noise variance is known, an minimumvariance parameter estimate can be obtained by choosing appropriate weights on the data [2]. However, numerous studies have been conducted, which clearly show that least

*Address correspondence to this author at the Test Center, College of Mechanical Engineering, Chongqing University, Chongqing, 400030, China; E-mail; Jzwy97@yahoo.com.cn squares estimators are vulnerable to the violation of these assumptions. Least squares uses the complete data and the outliers can not be eliminated. Sometimes even when the data contains only one bad datum, least squares estimates may be completely perturbed. To overcome the shortcoming, weighted least square(WLS), random sample consensus least square(RANSAC) and least median of squares(LmedS) have been used. For nonlinear model, nonlinear least square(NLS) can be used [3].

Unfortunately, for a dynamic biochemical pathway, the measured data is usually a sequential sequence and is uncompleted, it may be difficult to obtain large enough complete data to get the unbiased estimation with traditional parameter estimation methods. Then, sequential methods, like Kalman filter(KF), are natural choice for the problem. When the system is linear, Kalman filtering technique is equivalent of the least-squares technique, because Kalman gain is chosen to minimize the estimated state covariance. Due to the recursive nature of Kalman filter, it is more suitable to the problems where the measurements are available in a serial manner, and can take the data uncertainty into account, therefore, it can be used as a robust estimated method [3-5]. For a nonlinear dynamic model, nonlinear versions of Kalman filter can be used.

Up to now, it is still a problem to get the estimation of model parameters based on the sequential simulation [6], and only few works relative with parameter estimation of nonlinear ODEs used to model the biochemical dynamic pathway have been developed [7]. In other areas, the nonlinear versions of KF have been developed to solve the problems of state estimation and parameter estimation, such as hydrology [8], soil carbon [9], ecosystem [10], thermal conductivity [11], target directed dynamics [12], reservoir 
[13], structural dynamics [14], wave dynamics [15] etc. Although there are some applications of nonlinear versions of KF for parameter and state estimation of a biochemical system, it is just first step towards more sophisticated approach [7].

There are different methods which have been used to get the parameter estimates for a dynamic system [16, 17], such as joint state and parameter estimation method $[13,14,18$ $20]$, dual state and parameter estimation method [7, 21] and batch processing methods. In which, with dual/joint state and parameter estimation methods, the state and parameter estimates can be obtained simultaneously with sequential methods, and for batch processing methods, such as LS, all observations have been considered and make a penalty function minimize to get the parameter estimation. For a biochemical dynamic pathway, the general case is that the kinetic model structure is known but its parameters, kinetic rate and equilibrium constants, are unknown or poorly known, then have to be identified from time-series measurements of chemical species. Unfortunately, the measured data can only include partial states, which means that the unmeasured states and the unknown parameters should be estimated. The measurement and the system uncertainties are also the major challenge for the parameter and state estimators applied to the biochemical dynamic pathway $[22,23]$. Therefore, it is a natural choice to obtain the estimation of the states and parameters for a biochemical dynamic model simultaneously by using dual/joint methods.

Stefano Mariani applies dual/joint state and parameter estimation with EKF for identification of the interlaminar model and of the debonding surface(s) on the basis of freesurface measurements only [24]. By comparing the results of state and parameter estimation with dual/joint EKF in pseudo-experimental testing and actual experimental testing for noise-free and noisy environment, the authors give some conclusions about dual/joint state and parameter estimation, and think that dual EKF is better than joint EKF. However, the conclusions are obtained by comparing the results between different methods. Unfortunately, the results depend on the initial conditions, which may bring some randomicity. Literatures $[23,25]$ also points out the convergence of EKF may be relative with the initial estimation of state, correct noise assumption, linearization methods etc.. Furthermore, a biochemical dynamic pathway is always described by a set of continuous-time ODEs, the parameters to be estimated are the parameters of the continuous-time ODEs, which should be discretized firstly for numerical analysis. However, different discretization methods will bring different accuracy, which will also affect the accuracy of the last parameter estimation. A biochemical dynamic pathway is of different dynamic features from the system discussed in [24], then the experimental methods shown in [24] may not necessarily address the specific challenges posed by the biochemical dynamic pathway well. It is worth investigating the dual/joint state and parameter estimation with EKF for a biochemical dynamic pathway. With the same reasons, literatures $[16,18,20,21,26]$ just show that EKF is a bestknown and effective method for parameter estimation of dynamic systems. Especially, some literatures, such as [21], give some different results from [24], which shows that the performance of joint EKF may be better than that of dual
EKF. All of these also provide proofs to us that it is meaningful to study the methods for parameter estimation of a biochemical dynamic pathway.

In this paper, aim at the parameter estimation with incomplete measured data for a biochemical dynamic pathway, the validity of dual/joint state and parameter estimation methods with iterative EKF for the parameter identification of a biochemical dynamic pathway and the convergence of the two methods under different initial conditions for implementing EKF algorithm will be investigated. By this way, it can be found which one is better for parameter estimation of a biochemical dynamic pathway. In the following sections, the algorithm of parameter estimation with EKF is firstly given for a biochemical dynamic pathway; then the results with dual/joint EKF under different noisy measurement environment and initial conditions will be investigated to compare the effect between the two methods for parameter estimation of a biochemical dynamic pathway; at last, some conclusions on the two methods can be obtained.

\section{THE ALGORITHMS OF PARAMETER ESTIMATION WITH ITERATIVE EKF FOR A BIOCHEMICAL DYNAMIC PATHWAY}

\subsection{Biochemical Dynamic Pathway}

A biochemical dynamic pathway can be modeled as a nonlinear system described by a set of nonlinear ODEs. Linear ODEs only can be used to model an irreversible chain reaction [23]. Even for a linear model, when the parameters are regarded as unknown variables and estimated with the state simultaneously, just like what will be done in the paper later, the linear model will become a nonlinear model too. Furthermore the linear model is just a special case of a nonlinear model. So without loss of generality, the parameter estimation problem is investigated for a biochemical dynamic pathway modeled with a set of nonlinear ODEs. A dynamic signal pathway can be modeled with the following ODEs representation

$$
\begin{aligned}
& \dot{x}(t)=f(x(t), u(t), \theta)+w(t), \\
& z(t)=g(x(t), \theta)+v(t),
\end{aligned}
$$

Where equation (1) is the system dynamic model and equation (2) is the measurement model. $x \in R^{n}$ is the system states. $\theta \in R^{p}$ is the parameters of the model, for a biochemical dynamic pathway, which stands for reaction rates. $u \in R^{i}$ is the input, for a biochemical pathway, which represents external cellular signals, if only the intracellular biochemical pathway is studied, or the biochemical pathway is not subject to external cellular signals, it can be taken as $u=0 . f(\cdot)$ denotes linear or nonlinear functions which corresponding to the biochemical reactions; $g(\cdot)$ determines which states can be measured. For a simple case, it can be a linear model. $z \in R^{m}$ shows the measured states. $w$ is process noise error, whose covariance is $Q=E\left(w w^{T}\right)$ and $v$ is the measurement noise error, whose covariance is $R=E\left(v v^{T}\right)$. 
The continuous-time ODEs model should be discretized firstly for analysis purpose. Unfortunately, there is no general direct discretization mapping for nonlinear ODEs, therefore, other methods should be found to discretize the nonlinear continuous-time model. Literature [23] compares some one-step system discretization methods, such as Taylor-Carleman method, Monaco and Normand-Cyrot's method, and multi-step system discretization methods, such as Runge-Kutta method. From the comparison, it has shown that Runge-Kutta method can be a natural choice for discrete-time system representation. In numerical analysis, the Runge-Kutta methods are an important family of implicit and explicit iterative methods for the approximation of solutions of ODEs. It propagates a solution over an interval by combining the information from several Euler-style steps and then uses the information obtained to match a Taylor series expansion to some higher order [23, 27]

$$
x(k+1)=x(k)+h f(x(k)) \text {, }
$$

where $h$ is the sampling interval, $t_{k+1}=t_{k}+h$.

The discrete-time representation of continuous-time pathway model (1) and (2) can be rewritten as follows by using Runge-Kutta method:

$$
\begin{aligned}
& x(k+1)=x(k)+R(x(k))=F(x(k), \theta), \\
& z(k)=g(x(k), \theta)+v(k) .
\end{aligned}
$$

The commonly used method in the family of RungeKutta methods is often referred as "RK4" or simply as "the Runge-Kutta method", which is the fourth-order RungeKutta formula.

$$
R(x(k))=\left(k_{1}+2 k_{2}+2 k_{3}+k_{4}\right) / 6,
$$

where

$k_{1}=h f(x(k))$

$k_{2}=h f\left(x(k)+k_{1} / 2\right)$.

$k_{3}=h f\left(x(k)+k_{2} / 2\right)$

$k_{4}=h f\left(x(k)+k_{3}\right)$

Runge-Kutta method takes multi-step information, which enhances the discretization accuracy and reduces the complexity of mathematical expressions compared with that using higher-order derivative approximation in one-step discretization. Moreover, Runge-Kutta method could provide a general discrete-time ODE representation for either linear or nonlinear ODEs [23].

\subsection{Dual/Joint Parameter and State Estimation Algorithm with EKF}

With the discrete-time dynamic system model shown as equation (4), iterative extended Kalman filter(EKF) can be used to get the state and parameter estimation simultaneously $[28,29]$.

\section{A. EKF Algorithm}

Kalman filter is quite easy to be implemented and an optimal estimator for a linear system. However, as shown above, most of biochemical dynamic pathways are unfortunately nonlinear, and should be linearized for using
Kalman filter. Extended Kalman filter linearizes the nonlinear system by calculating the Jacobians of nonlinear functions for the dynamic system model (shown in the equation (4a)) and measurement model (equation (4b)) [3032]

$$
\begin{gathered}
A_{k}=\left.\frac{\partial F(x, \theta)}{\partial x}\right|_{\hat{x}_{k \mid k}, \theta}, \\
H_{k}=\left.\frac{\partial g(x)}{\partial x}\right|_{\hat{x}_{k \mid k-1}} .
\end{gathered}
$$

The Jacobians matrices $A_{k}, H_{k}$ will vary in time with the estimated state $\hat{x}_{k}$. Then the EKF can be implemented like a regular Kalman filter which includes two update:

(a) time update equations

$$
\begin{aligned}
& \hat{x}_{k \mid k-1}=F\left(\hat{x}_{k-1 \mid k-1}, \theta\right), \\
& \hat{P}_{k \mid k-1}=A_{k} \hat{P}_{k-1 \mid k-1} A_{k}^{T}+Q_{k} ;
\end{aligned}
$$

(b) measurement update equations

$$
\begin{aligned}
& \hat{x}_{k \mid k}=\hat{x}_{k \mid k-1}+K_{k}\left(z_{k}-g\left(\hat{x}_{k \mid k-1}\right)\right), \\
& K_{k}=\hat{P}_{k \mid k-1} H_{k}^{T}\left(H_{k} \hat{P}_{k \mid k-1} H_{k}^{T}+R_{k}\right)^{-1}, \\
& \hat{P}_{k \mid k}=\left(I-K_{k} H_{k}\right) \hat{P}_{k \mid k-1} .
\end{aligned}
$$

\section{B. Dual State and Parameter Estimation with EKF (DEKF)}

For a dual state and parameter estimation method, a separate state-space representation is used for the state and the parameters. Two EKFs are run simultaneously for state and parameter estimation respectively. At every time step, current parameter estimation is used in the state filter, and the current state estimate is used in the parameter filter, then the states and parameters can be estimated simultaneously. The algorithm for dual extended Kalman filter implementation is [21]

(a) time-update equations

For the parameter filter are

$\hat{\theta}_{k \mid k-1}=\hat{\theta}_{k-1 \mid k-1}$,

$\hat{P}_{\theta k \mid k-1}=\lambda^{-1} \hat{P}_{\theta k-1 \mid k-1}$

Where $\lambda \in\left(\begin{array}{ll}0 & 1]\end{array}\right.$ is referred as the "forgetting factor". In the simulation of this paper, it is taken as $\lambda=0.9999$.

For the state filter are

$\hat{x}_{k \mid k-1}=F\left(\hat{x}_{k-1 \mid k-1}, \hat{\theta}_{k \mid k-1}\right)$,

$\hat{P}_{x k \mid k-1}=A_{k} \hat{P}_{x k-1 \mid k-1} A_{k}^{T}+Q_{k} ;$

(b) measurement update equations

For the state filter are 
$\hat{x}_{k \mid k}=\hat{x}_{k \mid k-1}+K_{k}^{x}\left(z_{k}-g\left(\hat{x}_{k \mid k-1}\right)\right)$,

$K_{k}^{x}=\hat{P}_{x k \mid k-1} H_{k}^{T}\left(H_{k} \hat{P}_{x k \mid k-1} H_{k}^{T}+R_{k}\right)^{-1}$,

$\hat{P}_{x k \mid k}=\left(I-K_{k}^{x} H_{k}\right) \hat{P}_{x k \mid k-1}$;

And for the parameter filter are

$$
\hat{\theta}_{k \mid k}=\hat{\theta}_{k \mid k-1}+K_{k}^{\theta} e_{k}
$$

$K_{k}^{\theta}=\hat{P}_{\theta k \mid k-1}\left(C_{k}^{\theta}\right)^{T}\left(C_{k}^{\theta} \hat{P}_{\theta k \mid k-1}\left(C_{k}^{\theta}\right)^{T}+R_{k}^{\theta}\right)^{-1}$.

Where

$$
e_{k}=z_{k}-g\left(\hat{x}_{k \mid k-1}\right) \text {, }
$$

$$
C_{k}^{\theta}=-\frac{\partial e_{k}}{\partial \theta}=\frac{\partial g\left(\hat{x}_{k \mid k-1}\right)}{\partial \theta}=\left.H_{k} \frac{\partial \hat{x}_{k \mid k-1}}{\partial \theta}\right|_{\hat{\theta}_{k \mid k-1}} .
$$

\section{Joint State and Parameter Estimation with EKF (JEKF)}

In the joint state and parameter estimation method, the states and parameters are concatenated into a single joint state vector. By using sequential methods, like EKF, UKF, EnKF, the state and parameter estimation can be obtained simultaneously [18-20]. Therefore, firstly a new state vector must be constructed, which includes the states and unknown parameters to be estimated. Suppose the dimension of states $x_{s}$ is $n_{x}$ and that of unknown parameters $x_{\theta}$ is $n_{p}$, the new state vector can be written as

$$
x=\left(\begin{array}{c}
x_{s} \\
x_{\theta}
\end{array}\right),
$$

The dimension of the new state vector $x$ is $n=n_{x}+n_{p}$. After that the standard extended Kalman filter algorithm shown in the section (2.2) $A$ can be used to obtain the state and parameter estimates simultaneously.

\section{The Iterative Dual/Joint State and Parameter Algorithms}

The algorithm for dual/joint state and parameter estimation used in our simulations is described as the following:

\section{Step 1 Initialization}

Take the initial state and parameter estimation $\hat{x}_{0}$ and $\hat{\theta}_{0}$; the process noise covariance $\mathrm{Q}$ and measurement noise covariance R; the iterative times $N$; the length of observed data $\mathrm{L}=$ length(zobserve), where zobserve is the data vector of a measured state. In this paper, a small non-zero value is taken as process noise covariance corresponding to the estimated parameters that also includes the uncertainty in the parameters to be estimated;

\section{Step 2 Parameters Estimated with Iterative EKF}

(a) for dual state and parameter estimation approach for $\mathrm{j}=1: \mathrm{N}$ (iterative times)

if $\mathrm{j}==1 \theta_{0}=\hat{\theta}_{0}$; else $\theta_{0}$ should be the values of last step of pre-iteration; end
The initial state and parameter estimation error covariance are

$$
\hat{P}_{x 0}=\left(\hat{x}_{0}-x_{0} \text { true }\right)\left(\hat{x}_{0}-x_{0} \text { true }\right)^{T}
$$

and $\hat{P}_{\theta 0}=\left(\theta_{0}-\theta\right.$ true $)\left(\theta_{0}-\theta \text { true }\right)^{T}$ respectively;

In practice, the true values of states $x_{0}$ true and parameters Atrue may be not known, then the initial covariance could be taken by basing on the prior knowledge.

for $\mathrm{i}=1: \mathrm{L}$ (time series points of measured data)

Implementing the dual state and parameter estimation algorithm shown in section (2.2) $B$.

end of once iterative EKF

end of $\mathrm{N}$ times iterative $\mathrm{EKF}$

(b) for joint state and parameter estimation approach for $\mathrm{j}=1: \mathrm{N}$

if $\mathrm{j}==1 \theta_{0}=\hat{\theta_{0}}$; else $\theta_{0}$ should be the values of last step of pre-iteration; end

The initial joint state vector is $x_{0}=\left[\hat{x}_{0} \theta_{0}\right]$ and the true joint state vector is $x$ true $=\left[x_{0}\right.$ true $\theta$ true $]$;

The initial state estimation error covariance is $\hat{P}_{0}=\left(x_{0}-x\right.$ true $)\left(x_{0}-x \text { true }\right)^{T}$ or taken by basing on the prior knowledge.

for $\mathrm{i}=1: \mathrm{L}$

Implementing the joint state and parameter estimation algorithm shown in the section (2.2) $C$.

end of once iterative EKF

end of $\mathrm{N}$ times iterative EKF

Step 3 Take the values at the end of last iteration for JEKF and DEKF algorithm as the parameter estimates and the values of the last iteration as the state estimates for the biochemical dynamic pathway

Step4 Compute and compare the residual mean-square error(RMSE) of the state estimates between different methods and relative root squared error(RRSE) between the estimated parameters and true parameters.

\section{RESULTS AND DISCUSSION}

\section{Example: Simulation Results Illustration}

In this paper, Michaelis-Menten model is used as an example for nonlinear model stimulation. Within this framework, the conversion of the substrate $\mathrm{S}$ into the product $\mathrm{P}$ is catalyzed by the enzyme $\mathrm{E}$. The enzyme and the substrate form an intermediate enzyme-substrate complex ES which can degrade into the reactants or into product and enzyme. The kinetic mechanism can be presented in the graphical form [31]

$$
E+S \underset{k_{2}}{\stackrel{k_{1}}{\longrightarrow}} E S \stackrel{k_{3}}{\longrightarrow} E+P .
$$


Where the $k_{i}(\mathrm{i}=1,2,3)$ denote the rate coefficients for each elementary reaction. $x_{1}, x_{2}, x_{3}, x_{4}$ denote the concentrations of components E,S,ES,P, the kinetic rate equations for the pathway shown in the equation (23) are now a system of four coupled differential equations

$$
\dot{x}_{s}=\left[\begin{array}{l}
\dot{x}_{1} \\
\dot{x}_{2} \\
\dot{x}_{3} \\
\dot{x}_{4}
\end{array}\right]=\left[\begin{array}{l}
-k_{1} x_{1} x_{2}+k_{2} x_{3}+k_{3} x_{3} \\
-k_{1} x_{1} x_{2}+k_{2} x_{3} \\
k_{1} x_{1} x_{2}-k_{2} x_{3}-k_{3} x_{3} \\
k_{3} x_{3}
\end{array}\right]=f\left(x_{s}(t), \theta\right)
$$

Where $\theta=\left[\begin{array}{lll}k_{1} & k_{2} & k_{3}\end{array}\right]^{\prime}$ is unknown and time-invariable and will be estimated. Eq. (24) is the Michaelis-Menten system model which includes 4 states and 3 parameters. Suppose that only the change of concentration E, i.e. $\left[x_{1}\right]$ can be measured, and the measurement function is linear, then the measurement model ( $4 \mathrm{~b})$ can be rewritten as

$$
z(k)=H_{s} x_{s}(k)+v_{s}(k)
$$

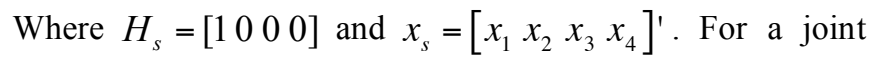
state and parameter estimation application of the model, a new single state vector including the four states and three parameters can be written as

$x=\left[\begin{array}{ll}x_{s} & \theta\end{array}\right]=\left[\begin{array}{lllllll}x_{1} & x_{2} & x_{3} & x_{4} & k_{1} & k_{2} & k_{3}\end{array}\right]^{\prime}$,

Then the continuous-time model can be expressed with the single state vector as:

$\dot{x}=\left[\begin{array}{l}\dot{x}_{s} \\ \dot{\theta}\end{array}\right]=\left[\begin{array}{c}f\left(x_{s}(t), \theta\right) \\ 0\end{array}\right]=f(x(t))$,

And $H_{s}$ should be changed into $H=\left[\begin{array}{lllllll}1 & 0 & 0 & 0 & 0 & 0 & 0\end{array}\right]$, the measurement model is

$$
z(k)=H x(k)+v(k) \text {. }
$$

Solving the ODEs denoted in the equation (24) with

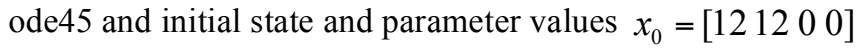
and $\theta=\left[\begin{array}{lll}0.18 & 0.02 & 0.23\end{array}\right]$, the output of the states can be used as the true state output. Because only the change of the concentration of $\mathrm{E}$ can be measured, the measured data can be obtained by adding different normal noise on the true data, zobserve $=\mathrm{z}+\operatorname{sqrt}(\mathrm{R}) * \operatorname{randn}(\operatorname{size}(\mathrm{z}))$ for the following simulation, where $\mathrm{z}$ is the output [E] of ODE solvers. In the following, the algorithms described in the 2.2(D) are used to obtain the parameter estimation. The values used to solve ODEs are taken as the true values of the initial state and parameters,$\quad$ i.e. suppose

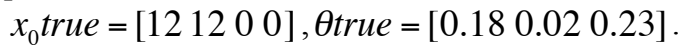

In the following, we will investigate the results of parameter and state estimation with DEKF and JEKF under different initial conditions and different noisy environment for Michaelis-Menten Model.

\section{A. Under the Weak Measurement Noisy Environment}

(a) The measurement data with noise covariance $\mathrm{R}=0.01$, initial state estimation is $\hat{x}_{0}=\left[\begin{array}{llll}10 & 10 & 0.2 & 0.2\end{array}\right]$, process noise covariance is $\mathrm{Q}=1 \mathrm{e} 0 * \mathrm{eye}(4)$, initial state estimation covariance is $\hat{P}_{x 0}=\left(\hat{x}_{0}-x_{0}\right.$ true $)\left(\hat{x}_{0}-x_{0} \text { true }\right)^{T}$, and the true parameters is $\theta$ true. By implementing EKF algorithm shown in (2.2)A, we can get the state estimates with true parameters shown in the figure 1(b)(with dot line).

Then it is supposed that the initial parameter estimation is $\hat{\theta}_{0}=\left[\begin{array}{lll}0.2 & 0.2 & 0.2\end{array}\right]$, the iteration times is $\mathrm{N}=100$. With the DEKF and JEKF algorithms, the parameter estimates of the model are shown in the Fig. (1a). the state estimates at the last iteration with DEKF and JEKF are also shown in the Fig. (1b). In the figure, dash dot lines denote the state estimation with JEKF, dashed lines denote the state estimation with DEKF and dot lines denote the state estimation with standard EKF and the true parameters.

The root mean-square error(RMSE) of state estimation is defined as eq. (29). The comparison for the RMSE of the last iteration with DEKF and JEKF and that of standard EKF with true parameters are shown in the Table 1a. For showing the estimation error of the parameters at every sampled time step, the relative root squared error(RRSE) (shown in the eq. (30)) is introduced to evaluate the error of parameter estimation.

$$
\begin{aligned}
& \text { RMSE }=\sqrt{\sum_{i=1}^{L}\left(\hat{x}_{i}-x \text { true }\right)^{2} / L}, \\
& R R S E=\frac{\left\|\theta^{*}-\hat{\theta}_{i k}\right\| .}{\left\|\theta^{*}\right\|} .
\end{aligned}
$$

Where $\theta^{*}$ denotes the true parameters and $\hat{\theta}_{i k}$ denotes the estimated parameter at a time step $\mathrm{k}$ in the $\mathrm{i}^{\text {th }}$ iteration. Table (1b) shows the last estimated parameters and their RRSE at the end of last iteration with different methods.

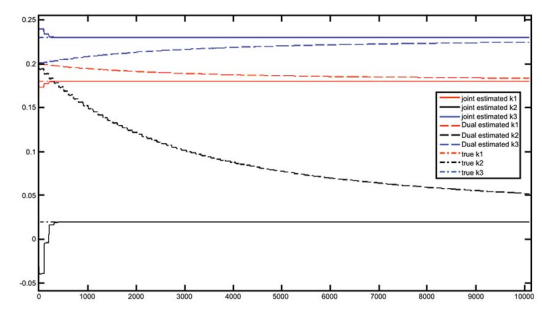

(a)

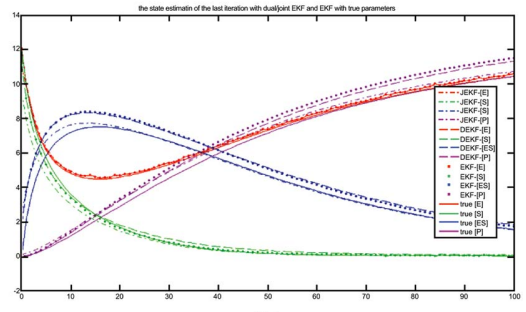

(b)

Fig. (1). The output of estimated parameters and states with different methods and initial conditions (a). 
Table (1a). The RMSE of State Estimates at the Last Iteration with DEKF, JEKF and EKF

\begin{tabular}{|c|c|c|c|}
\hline RMSE & with EKF & with DEKF & with JEKF \\
\hline \hline$[\mathrm{E}]$ & 0.10877 & 0.10876 & 0.1486 \\
\hline$[\mathrm{S}]$ & 0.16759 & 0.20895 & 0.46217 \\
\hline$[\mathrm{ES}]$ & 0.62001 & 0.64248 & 0.4059 \\
\hline$[\mathrm{P}]$ & 0.75685 & 0.59619 & 0.33003 \\
\hline Total & 1.6532 & 1.5564 & 1.3467 \\
\hline
\end{tabular}

Table (1b). Estimated Parameters with Different Methods

\begin{tabular}{|c|c|c|c|}
\hline Parameters & k1 & k2 & k3 \\
\hline \hline True & 0.18 & 0.02 & 0.23 \\
\hline Initial & 1.0 & 0.3 & 0.8 \\
\hline DEKF/RRSE & $0.1836 / 0.0199$ & $0.0522 / 1.6084$ & $0.2246 / 0.0233$ \\
\hline JEKF/RRSE & $0.1801 / 0.0003$ & $0.0200 / 0.0011$ & $0.2300 / 0.0001$ \\
\hline
\end{tabular}

(b) Change the initial state and parameter estimations to $\hat{x}_{0}=\left[\begin{array}{llll}5 & 5 & 5 & 5\end{array}\right], \hat{\theta}_{0}=\left[\begin{array}{lll}0.5 & 0.5 & 0.5\end{array}\right]$ and keep other initial conditions in (a) unchanged, implementing the algorithms again, the results are shown in the Fig. (2) and Table 2 respectively.

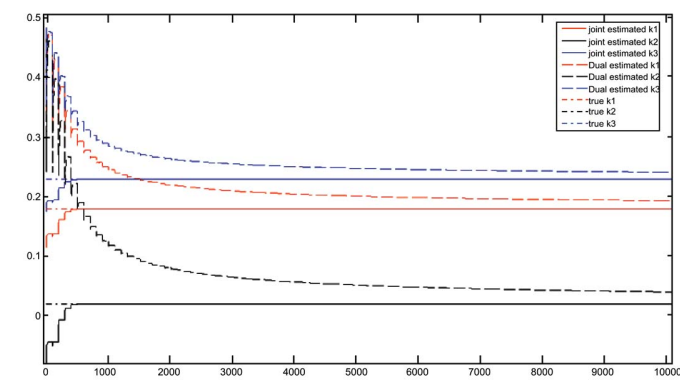

Fig. (2). The parameter estimation with different methods and initial conditions (b).

Table 2. Estimated Parameters with Different Methods

\begin{tabular}{|c|c|c|c|}
\hline Parameters & K1 & K2 & K3 \\
\hline \hline True & 0.18 & 0.02 & 0.23 \\
\hline Initial & 1.0 & 0.3 & 0.8 \\
\hline DEKF/RRSE & $0.1933 / 0.0740$ & $0.0400 / 0.9996$ & $0.2412 / 0.0489$ \\
\hline JEKF/RRSE & $0.1801 / 0.0003$ & $0.0200 / 0.0011$ & $0.2300 / 0.0001$ \\
\hline
\end{tabular}

Compare Fig. (1) with Fig. (2) and Table 1 with Table 2, it can be found that with different initial conditions, the parameter estimates with DEKF change significantly and they still have not converged to the true parameters after 100 iterations, while those with JEKF keep almost same accuracy and converge to the true parameters at about $5^{\text {th }}$ iteration. That is to say, with JEKF, we can obtain more robust and accurate parameter estimation than with DEKF. From Table 1a, an amazing result can also be found. Though true parameters are applied when the standard EKF algorithm is implemented, the more accurate state estimates with JEKF and DEKF can be obtained. For the results, we think the explanation may be that for a nonlinear system, the EKF algorithm itself is an approximate estimation algorithm, while the dual/joint EKF also learns an approximate model, which could be better matched to the state estimation approximation [21]. Furthermore, JEKF and DEKF use iterative EKF algorithm, which is less sensitive to the choice of the filter's parameters(such as process and measurement noise covariance, initial state estimation etc.) than standard EKF [33].

\section{B. The Noisy Measurement Environment}

The validity of DEKF and JEKF is investigated further for parameter estimation of a biochemical dynamic pathway under different initial conditions and different noisy measurement data.

(c) The measured data has been contaminated by noise badly, the measurement noise covariance is $\mathrm{R}=10$, the initial state and parameter estimation are $\hat{x}_{0}=\left[\begin{array}{llll}10 & 10 & 0.2 & 0.2\end{array}\right]$ and $\hat{\theta}_{0}=\left[\begin{array}{lll}1.0 & 0.3 & 0.8\end{array}\right]$, the iteration time still is $\mathrm{N}=100$, process noise covariance is $\mathrm{Q}=1 \mathrm{e}-3 * \operatorname{eye}(4)$, the initial state estimation covariance is $\hat{P}_{x 0}=\left(\hat{x}_{0}-x_{0}\right.$ true $)\left(\hat{x}_{0}-x_{0} \text { true }\right)^{T}$. The results are shown in the Fig. (3) and Table 3.

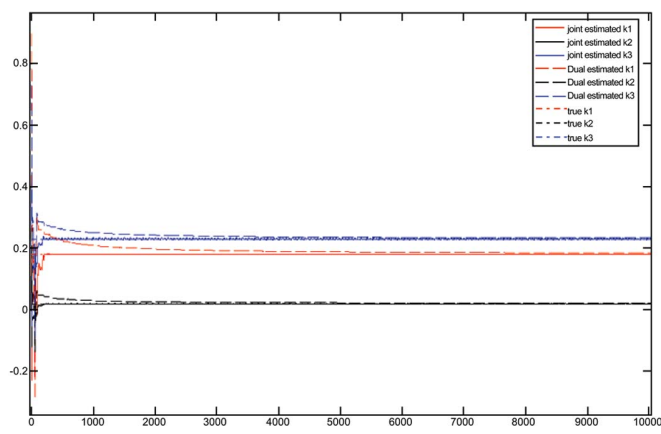

Fig. (3). The parameter estimation with different methods and initial conditions (c).

Table 3. Estimated Parameters with Different Methods

\begin{tabular}{|c|c|c|c|}
\hline Parameters & k1 & k2 & k3 \\
\hline \hline True & 0.18 & 0.02 & 0.23 \\
\hline Initial & 0.1 & 0.3 & 0.8 \\
\hline DEKF/RRSE & $0.1843 / 0.0237$ & $0.0215 / 0.0727$ & $0.2330 / 0.0129$ \\
\hline JEKF/RRSE & $0.1798 / 0.0008$ & $0.0199 / 0.0071$ & $0.2307 / 0.0031$ \\
\hline
\end{tabular}

(d) Keep other initial conditions in (c) unchanged, change the process noise covariance to $\mathrm{Q}=1 \mathrm{e} 0 *$ eye $(4)$. The results are shown in the Fig. (4) and Table 4. 


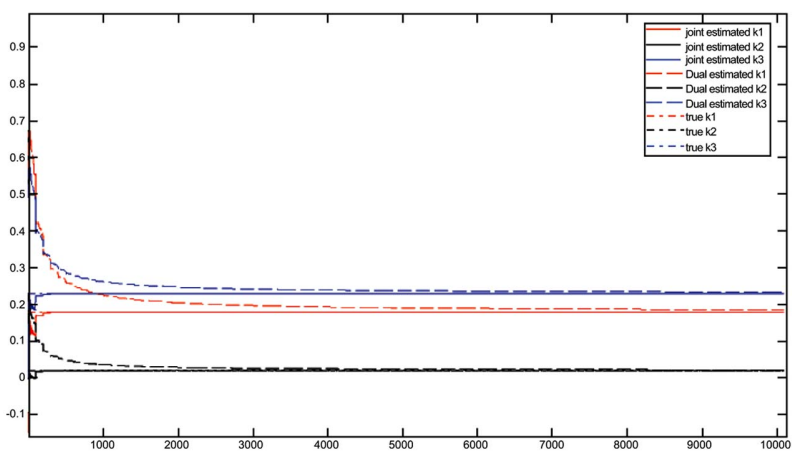

Fig. (4). The parameter estimation with different methods and initial conditions $(\mathbf{d})$.

Table 4. Estimated Parameters with Different Methods

\begin{tabular}{|c|c|c|c|}
\hline Parameters & $\mathbf{k 1}$ & $\mathbf{k 2}$ & $\mathbf{k 3}$ \\
\hline \hline True & 0.18 & 0.02 & 0.23 \\
\hline Initial & 1.0 & 0.3 & 0.8 \\
\hline DEKF/RRSE & $0.1854 / 0.0298$ & $0.0218 / 0.0915$ & $0.2337 / 0.0162$ \\
\hline JEKF/RRSE & $0.1794 / 0.0036$ & $0.0202 / 0.0101$ & $0.2300 / 0.0002$ \\
\hline
\end{tabular}

(e) Keep other initial conditions in (d) unchanged, change

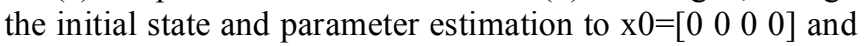
$\theta_{0}=\left[\begin{array}{lll}0.5 & 0.5 & 0.5\end{array}\right]$. The results are shown in the Fig. (5) and Table 5.

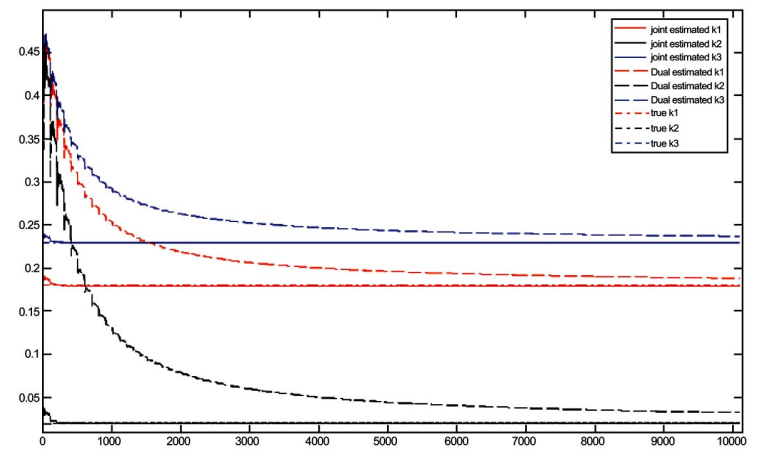

Fig. (5). The parameter estimation with different methods and initial conditions (e).

Table 5. Estimated Parameters with Different Methods

\begin{tabular}{|c|c|c|c|}
\hline Parameters & K1 & K2 & K3 \\
\hline \hline True & 0.18 & 0.02 & 0.23 \\
\hline Initial & 0.5 & 0.5 & 0.5 \\
\hline DEKF/RRSE & $0.1884 / 0.0467$ & $0.0326 / 0.6309$ & $0.2371 / 0.0309$ \\
\hline JEKF/RRSE & $0.1794 / 0.0036$ & $0.0202 / 0.0101$ & $0.2300 / 0.0002$ \\
\hline
\end{tabular}

By comparing Figs. $(\mathbf{3}, \mathbf{4 , 5})$ and Tables $\mathbf{3 , 4 , 5}$, we still can get the conclusions which are same as that in the weak noisy environment. Furthermore, when large enough measurement noise is taken, the DEKF and JEKF algorithms can keep more robust for different initial state estimation and process noise covariance. From the cases (c)(d)(e), it can be seen that the parameter estimations with different methods almost keep the same accuracy. However, the accuracy of parameter estimates with JEKF is still better than that with DEKF, furthermore, parameter estimation with JEKF is of faster convergence. All these cases show that JEKF is more robust than DEKF for parameter estimation of a biochemical dynamic pathway. By the way, the time implementing JEKF is shorter than that of DEKF. Use Sony laptop with Intel T2600(2.16GHz), 1GB DDR2 memory, the initial conditions in the case (e), the 100 iteration times, and 101 measured data points, the time for JEKF is $4.5160 \mathrm{~s}$, while that for DEKF is $5.4690 \mathrm{~s}$.

We have shown the validity of joint/dual state and parameter estimation with iterative EKF for the parameter estimation of the biochemical dynamic pathway, however, due to the limitation of EKF, such as first-order approximation which may lead to suboptimal and even divergence of the filter [6], the difficulty of implementing it because the Jacobian matrix should be computed to linearize the nonlinear system [34], it is difficult to be used for high dimension and complex biochemical dynamic pathways to get the state and parameter estimation simultaneously.

\section{FURTHER DISCUSSION}

To show whether the joint method with iterative EKF is effective for large biochemical dynamic pathways or not, the method is investigated further for NF-кB pathway .

The nuclear factor $\kappa \mathrm{B}(\mathrm{NF}-\kappa \mathrm{B})$ signaling pathway (see Fig. 6) is an important cellular signaling pathway, of which protein phosphorylation is a major factor controlling the activation of further downstream events [34-36]. The pathway includes 26 reaction species participating in 64 reactions. Out of the 26 reaction species, 24 species are changing dynamically and their concentrations are defined as the state variables.

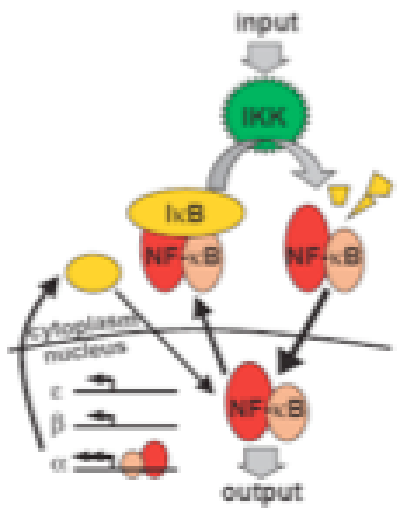

Fig. (6). IxB-NF-kB signal pathway module.

If the dynamic behavior of a biochemical signaling pathway is highly dependent on the values of some of the parameters, then these parameters are essential for the accuracy and reliablity of the biochemical dynamic model. Therefore it is important to estimate them accurately. 
Literature [26] has studied the sensitivity of the parameters and shown that parameters $\mathrm{k} 9, \mathrm{k} 28, \mathrm{k} 29, \mathrm{k} 36, \mathrm{k} 38$ and $\mathrm{k} 52$ are key parameters which capture the essential dynamics of the signaling pathway. We suppose these parameters are unknown and should be estimated, other parameters are known. Use the joint state and parameter estimation approach, so the new state vector is $x=\left(x_{s} x_{p}\right)$, whose dimensions is $n=n_{x}+n_{p}=24+6=30$.

The ODEs model for the pathway can be obtained from the literature [26]. By using the iterative JEKF algorithm shown in the section 2.2(C) and (D), the state and parameter estimation can be obtained.

The true initial state values are $\mathrm{x} 0$ true $=\left[\begin{array}{lll}0.1902 & 0.0003\end{array}\right.$ $\begin{array}{lllllllllllll}0.0821 & 0.0214 & 0.0091 & 0.0153 & 0.0065 & 0 & 0 & 0.1 & 0 & 0 & 0 & 0 & 0.0002\end{array}$ $\begin{array}{lllllllllll}0.1931 & 0.0014 & 0.0219 & 0.0003 & 0.0156 & 0.0002 & 0.0055 & 0.0006\end{array}$ $0.0005]$;

The true values of parameter $\mathrm{k} 9, \mathrm{k} 28, \mathrm{k} 29, \mathrm{k} 36, \mathrm{k} 38$ and k52 are $\theta$ true $=\left[\begin{array}{llllll}1.221 & 1.02713 & 0.0168 & 0.2448 & 0.018 & 11.1\end{array}\right]$;

Suppose the initial state estimation is $\hat{x}_{0}=$ $\mathrm{x} 0$ true $+0.001 * \exp (0.3 * \operatorname{randn}(1,24))$; the initial parameter estimation is $\theta_{0}=\theta t r u e+0.001 * \exp (0.3 * \operatorname{randn}(1,6))$; $\mathrm{Q}=[1 \mathrm{e}-$ $4 *$ eye(24) zeros(24,6); zeros(6,24) 1e-6*eye(6)]; $\mathrm{R}=10$, which is a little larger than the actual measurement noise. Iterative times $\mathrm{N}=30$.

Unfortunately, the simulation results show that some of the estimates have divergence with the algorithm. It also can be found that it is difficult to take right initial conditions to implement the iterative JEKF algorithms for large biochemical pathways and keep reliable with the increasing nonlinearity. The implementing of the iterative EKF algorithm becomes more sensitivity to the initial conditions and is difficult to tune due to the limitation of the algorithm itself. Therefore, it is difficult to be used for large and complex biochemical dynamic pathway to get the state and parameter estimation simultaneously. Instead, unscented Kalman filter(UKF) [20, 37], ensemble Kalman filter(EnKF) $[8,10]$, even particle filter(PF) $[38,39]$ could be used to solve the parameter estimation problem for complex and large biochemical dynamic pathways, which are also the subject of our future work.

\section{CONCLUSIONS}

With iterative EKF algorithm, the state and parameter estimation can be obtained for a biochemical dynamic pathway simultaneously by taking dual/joint state and parameter estimation approaches. However, it can be seen that with joint state and parameter estimation approach, more accurate and robust parameter estimates can be obtained and the estimated parameters converge to the true parameters faster, while the convergence of estimated parameters with dual state and parameter estimation method could be very slow under some initial conditions. It is also noticed that when large enough measurement noise is taken and the SNR is in a concessional level, the output of parameter estimation with the two approaches become robust for different initial state and process noise covariance estimation. It also can be found that the inherent linearization of extended Kalman filter can limit its applicability to biochemical pathways, e.g. when the states undergo large variations. And its applicability can be improved by choosing a small initial value of the covariance matrix to eliminate the possible inadvertent reliance on an unreliable tuning $[4,40]$.

\section{REFERENCES}

[1] D. Feng, S. Huang, Z.Wang, and D. Ho, "An unbiased parametric imaging algorithm for non-uniformly sampled biomedical system parameter estimation", IEEE Transactions on Medical Imaging, vol.15, no.4, 512-518, 1996.

[2] J. Kim, D. Bates, I. Postlethwaite, P. Heslop-Harrison and K.H. Cho, "Least-squares methods for identifying biochemical regulatory networks from noisy measurements". BMC Bioinformatics, vol. 8, p. 8, 2007.

[3] Z. Zhang, "Parameter Estimation Techniques: A tutorial with application to conic fitting"'. Image and Vision Computing Journal, vol.15, no.1, pp. 59-76, 1997.

[4] D. Dochain, "State and parameter estimation in chemical and biochemical processes: a tutorial", Journal of Process Control, vol. 13, pp. 801-818, 2003.

[5] M. Varziri, "Parameter estimation in nonlinear continuous time dynamic models with modeling errors and process disturbances", $\mathrm{PhD}$ thesis 2008: Queen's University, Kingston, Ontario, Canada

[6] O. Cappe and E.Moulines, "On the use of particle filtering for maximum likelihood parameter estimation", http://www.tsi.enst.fr/ cappe/

[7] J.D. Annan1, D.J. Lunt2, J.C. Hargreaves1, and P.J. Valdes, "Parameter estimation in an atmospheric GCM using the Ensemble Kalman Filter", Nonlinear Processes in Geophysics, vol. 12, pp. 363-371, 2005.

[8] M. Quach, N. Brunel and F. d'Alche-Buc. "Estimating parameters and hidden variables in non-linear state-space models based on ODEs for biological networks inference", Bioinformatics, vol.23, no.23, pp.3209-3216, 2007.

[9] H. oradkhani, S. rooshian, H.V. Gupta, and P.R. Houser, "Dual state-parameter estimation of hydrological models using ensemble Kalman filter', Advances in Water Resources, vol. 28, pp.135147, 2005.

[10] J. Koo, W.M. Bostick, J.W. Jones, A.J. Gijsman, and J.B. Naab, "Estimating soil carbon in agriculture systems using ensemble Kalman filter and DSSAT”, ASAE Annual Meeting 2003.

[11] A. Aksoy, F. Zhang, J.W. Nielsen-Gammon, and C. Epifanio, "Simultaneous state and parameter estimation with an ensemble Kalman Filter for thermally driven circulations. Part I: Experiments with Perfect Parameters", Journal of Geophysical Research, 2004

[12] R.Togneri, J. Ma, and L. Deng, "Parameter estimation of a targetdirected dynamic system model with switching states", Signal Processing, vol. 81, pp. 975-987, 2001.

[13] J.P. Jensen, "Ensemble Kalman Filtering for state and parameter estimation on a reservoir model. Master dissertation: Engineering Cybernetics", Norwegian University of Science and Technology, 2007.

[14] A. Corigliano , and S. Mariani, "Parameter identification in explicit structural dynamics: performance of the extended Kalman filter", Computer Methods in Applied Mechanics and Engineering, vol. 193, pp. 3807-3835, 2004.

[15] J. Kao, B. Williams, D. Higdon, and C. Huang, "Parameter estimation and uncertainty quantification for shock-wave simulations with a Bayesian inference approach", Geophysical Research Abstracts, vol. 8, p. 01341, 2006

[16] Z.W. Gao and D.W.C. Ho, "Proportional multiple-integral observer design for descriptor systems with measurement output disturbances", IEEE Proceedings of Control Theory Applications, vol.151, no.3, pp.279-288, 2004.

[17] Z.W. Gao, and H Wang, "Descriptor observer approaches for multivariable systems with measurement noises and application in fault detection and diagnosis", Systems and Control Letters, vol. 55, no. 4, pp. 304-313, 2006.

[18] M.N Bandyopadhyay, R.N Sharma, and R. Prakash, "Extended Kalman Filter approach to joint state and parameter estimation", IE(I) Journal-ID, Vol.84, Nov. 2003: 19-23

[19] R.V. der Merwe and E.A. Wan, "The square-root unscented Kalman Filter for state and parameter estimation. Proceedings for 
IEEE International Conference on Acoustics", Speech, and Signal Processing 2001, vol.6, pp. 3461-3464

[20] C.M. Trudinger, M.R. Raupach, P.J. Rayner and I.G. Enting, "Using the Kalman Filter for parameter estimation in biogeochemical models". Environmetrics, vol. 8, no. 19, pp. 849870, 2008, www.interscience.wiley.com [Accessed on 14 Feb. 2008]

[21] S. Haykin, Kalman Filtering and Neural Network, John Wiley \& Sons Inc., New York, USA, 2001.

[22] J.L. Willems, and F.M. Callier, "Divergence of the stationary Kalman filter for correct and for incorrect noise variances", IMA Journal of Mathematical Control \& Information, vol. 9, pp. 47-54, 1992.

[23] F. He, L. Yeung and M. Brown, "Discrete-time model representation for biochemical pathway systems", IAENG International Journal of Computer Science, 34:1, IJCS_34_1_15, 2007.

[24] S. Mariani, and A. Corigliano, "Impact induced composite delamination: state and parameter identification via joint and dual extended Kalman filters", Computer Methods in Applied Mechanics and Engineering, vol. 194, pp. 5242-5272, 2005.

[25] L. Ljung, "Asymptotic behavior of the extended Kalman Filter as a parameter estimator for linear systems", IEEE Transaction on Automatic Control, vol. 24, no.1, pp. 36-50, 1979.

[26] H. Yue, M. Brown, J. Knowles, H. Wang, B. David S. Kell, and B. Douglas, "Insights into the behavior of systems biology models from dynamic sensitivity and identifiability analysis: a case study of an NF-кB signaling pathway", Molecular BioSystems, vol. 2, no.12, pp. 640-649, 2006.

[27] http://en.wikipedia.org/wiki/Runge_kutta, Runge-Kutta methods.

[28] Z. Gao, X Dai, T. Breikin, and H. Wang, High-gain observer-based parameter identification with application in a gas turbine engine, Proceedings of the 17th World Congress, Seoul, Korea, 2008, pp. $1408-1413$.

[29] Z.W. Gao, "PD observer parameterization design for descriptor systems", Journal of the Franklin Institute, vol. 342, no.5, pp. 551564,2005
[30] G. Welch, and G. Bishop. "An introduction to the Kalman Filter. http://info.acm.org/pubs/toc/CRnotice.html

[31] O. Wolkenhauer. Systems Biology: Dynamic Pathway Modeling. www.sbi.uni-rostock.de, December, 2006:62

[32] S.J. Julier and J.K. Uhlmann, "A new extension of the Kalman Filter to nonlinear systems," in Proc. of AeroSense: The 11th Int Symp. on Aerospace/Defence Sensing, Simulation and Controls, 1997.

[33] S. Azad, and J.S. Farrokh. "Sensitivity Analysis of EKF and Iterated EKF Pose Estimation for Position-Based Visual Servoing", Proceedings of the 2005 IEEE Conference on Control Applications, Toronto, Canada, August 28-31, 2005, pp. 755-760.

[34] B. Tian, D.E. Nowak, M. Jamaluddin, S. Wang and A.R. Brasier, The Journal of Biological Chemistry, vol. 280, pp. 17435-17438, 2005.

[35] N.D. Perkins and T.D. Gilmore, "Good cop. The deifferent faces of NF-KB', Cell Death and Differentiation”, vol.13, pp. 759-772, 2006.

[36] A. Hoffmann, A. Levchenko, M.L. Scott and D. Baltimore, "The IkB-NF-kB signaling module: temporal control and selective gene activation", Science, vol. 298, pp. 1241-1245, 2002.

[37] E.A. Wan and R. der Merwe, "The unscented Kalman Filter for nonlinear estimation. Adaptive systems for signal processing, communications, and control symposium 2000. AS-SPCC. Lake Louise, Alta., Canada, October, 2000, pp. 153-158

[38] Estimation of state of nonlinear systems using a particle filter. http://www.ualberta.ca/ slshah

[39] F. Ababsa, M. Mallem and D. Roussel, "Comparison between particle filter approach and Kalman Filter-Based technique for head tracking in augmented reality systems", Proceedings of the 2004 IEEE international Conference on Robotics \& Automation, New Orleans, LA, 2004, pp. 1021-1026.

[40] K. Reif, S. Gunther, E. YazStefan, and R. Unbehaven, "Stochastic stability of the discrete-time extended Kalman Filter', IEEE Transactions On Automatic Control, vol. 44, no. 4, pp. 714-728, 1999.

(C) Ji and Brown; Licensee Bentham Open.

This is an open access article licensed under the terms of the Creative Commons Attribution Non-Commercial License (http://creativecommons.org/licenses/by-nc/3.0/) which permits unrestricted, non-commercial use, distribution and reproduction in any medium, provided the work is properly cited. 